\title{
Fewer Grammatical Errors Means Less Editing
}

\author{
Mark B. Mycyk, MD
}

Boston Medical Center, Boston, MA and Toxikon Consortium, Chicago, IL

\begin{abstract}
The words "fewer" and "less" are commonly confused and misused in everyday speech and in writing. Use the word "fewer" when items can be counted individually; use "less" when a quantity cannot be counted.
\end{abstract}

Incorrect: If less than two tablets were ingested, the poison center recommends keeping the child at home.

Correct: If fewer than two tablets were ingested, the poison center recommends keeping the child at home.
A related error involves misuse of the words "number" and "amount." Use the word "number" when something can be counted individually; use "amount" when something cannot be counted.

Example \#1 (correct): In the resuscitation room, a large number of tablets were evacuated from the patient's stomach during the gastric lavage procedure.

Example \#2 (correct): A small amount of green fluid remained in the bottle of antifreeze found next to the patient's bed. 